\title{
O conceito de integral de Riemann do ponto de vista da congruência semântica
}

\section{The concept of Riemann's integral from the viewpoint of semantic congruence}

\author{
Claudete Cargnin \\ claucf@gmail.com \\ Rui Marcos de Oliveira Barros \\ rmobarros@uem.br
}

\begin{abstract}
Resumo
Este artigo discute a congruência semântica entre representações de conceitos componentes do conceito de Integral de Riemann e o papel da conversão na sua compreensão. São feitas análises de congruência, baseadas nos critérios estabelecidos por Duval, para a notação de somatório, o conceito de convergência e o cálculo de área de uma região limitada mediante integral definida. Conclui-se que o conceito de Integral de Riemann está repleto de não congruências em sua composição e requer maior atenção e cuidado ao ser introduzido aos alunos de Cálculo I.
\end{abstract}

Palavras-Chave: Congruência Semântica; Integral de Riemann; Cálculo I; Notação de somatório; Convergência; Área.

\begin{abstract}
This article discusses the semantic congruence between representations of concepts components of the concept of Riemann integral and the role of conversion in their understanding. Analyses are made of congruence, based on criteria established by Duval, for the summation notation, the concept of convergence and the calculation of the area of a region bounded by definite integral. We conclude that the concept of Riemann integral is full of non-congruence in their composition and requires more attention and care to be introduced to students in Calculus I.
\end{abstract}

Keywords: Semantic Congruence; Riemann integral; Calculus I ; Summation Notation ; Convergence ; Area.

\section{Introdução:}

Há algumas décadas vem se intensificando o estudo em torno da dificuldade de aprendizagem dos conceitos estudados em Cálculo I. Alternativas metodológicas como o uso de recursos computacionais, trabalhos em grupo, uso da língua natural tem sido apontadas.

Nos últimos anos tem se intensificado as pesquisas acadêmicas em torno das integrais. Em particular, as pesquisas de Melo (2002) e Barroso et al (2008) investigaram a possibilidade de construção do conceito de integral de Riemann tendo os recursos computacionais como suporte. Ambas as pesquisas indicam que o uso adequado de ferramentas computacionais contribui para a compreensão do conceito de integral definida como limite de uma soma de Riemann.

Apesar das investigações, ainda é alto o índice de reprovação em Cálculo I. Uma das possibilidades é a negligência, por parte de livros didáticos e professores, das dificuldades 
inerentes à própria matemática. Muitos conceitos estudados, como o de integral de Riemann (integral definida), por exemplo, estão carregados de significação e exigem um tratamento distinto dos conceitos referentes a outras áreas de conhecimento.

Em particular, o conceito de integral definida envolve vários outros conceitos, como o de somatório e convergência, que traz suas dificuldades próprias e que aumentam a complexidade do conceito que é objeto de estudo, nesse caso o de integral definida.

Duval (2009) estabelece que, para o desenvolvimento de uma atividade matemática, é preciso que os sistemas semióticos utilizados permitam o cumprimento de três atividades cognitivas fundamentais, ligadas à semiósis: a comunicação, o tratamento e a objetivação.

A atividade cognitiva de comunicação permite que um conjunto de traços se torne uma representação de um objeto dentro de um sistema semiótico. Já a atividade de tratamento permite transformar representações em outras representações dentro do mesmo sistema semiótico, levando em conta apenas as regras próprias do sistema. A atividade de objetivação ou tomada de conhecimento permite que uma representação em um sistema semiótico seja convertida em outra representação em outro sistema semiótico.

É justamente essa conversão de representações que deve ser adequadamente trabalhada na educação escolar, a fim de que o aluno possa verdadeiramente acessar o objeto matemático em estudo. Entretanto, ela não é simples. A conversão não é natural para a maior parte dos alunos. Ela requer a coordenação de diferentes sistemas semióticos, mas, para isso, é preciso uma exploração mais consistente, no ensino, das diferentes representações. Além disso, a conversão ainda exige a percepção da diferença entre representante e representado, entre sentido e referência.

Nesse artigo, busca-se analisar, à luz da Teoria do Registro de Representação Semiótica, as dificuldades envolvidas para a compreensão do conceito de integral definida, dado pela igualdade (1):

$$
\int_{a}^{b} f(x) d x=\lim _{n \rightarrow \infty} \sum_{i=1}^{n} f\left(x_{i}\right) \cdot\left(\frac{b-a}{i}\right)
$$

De modo geral, (1) é tratada de maneira simplista em alguns livros didáticos, que desprezam a complexidade envolvida em cada componente dessa expressão.

Um olhar mais atento para (1) nos indica que sua compreensão requer a compreensão dos seguintes aspectos:

- A notação de somatório;

- A convergência de sequências e séries; 
- O cálculo da área de uma região mediante soma de infinitas áreas de retângulos e da integral definida.

Cada um desses aspectos traz consigo dificuldades significativas, em termos de congruência semântica, que, ao serem unidos numa única expressão, tornam-na ainda mais complexa. São algumas dessas análises que nos propomos a apresentar nesse artigo.

Admitimos que é a operação de conversão de representações que viabiliza a diferenciação representante $\mathrm{X}$ representado, e, embora menosprezada pelo ensino, deve ser cautelosamente analisada, a fim de compreender as reais dificuldades do aluno para determinar um modo de atuação sobre elas.

\section{A congruência semântica e a dificuldade de conversão}

Duval (2009) associa três fenômenos ao desenvolvimento do conhecimento, relativos à semiósis e à operação de conversão, isto é, ao acesso ao objeto representado. São eles:

1. Diversificação dos registros de representação semiótica. Cada tipo de registro tem ênfase em questões de aprendizagens específicas, por isso há necessidade de diversificação, de buscar a complementaridade das informações relativas ao objeto representado, como aponta Moretti (2002);

2. Diferenciação entre representante e representado (ou forma e conteúdo): está associada à compreensão de que uma representação apenas representa o objeto, não é o objeto. A ele é possível associar outras representações;

3. Coordenação entre os diferentes registros: assume-se que quando o indivíduo consegue facilmente mudar a forma de representação do objeto, ele compreendeu o objeto.

Independente do nível de ensino, básico ou superior, é importante que o professor busque atender a esses aspectos. Isso pode ser feito mediante uso de tarefas de produção e compreensão. As tarefas de produção mobilizam as atividades cognitivas de formação/comunicação de representação num registro semiótico (correspondente à função discursiva de designação de objetos) e de tratamento. As tarefas de compreensão mobilizam as atividades cognitivas de formação e conversão ou as três atividades simultaneamente: formação, tratamento e conversão.

Para compreendermos um pouco melhor os aspectos que influenciam na conversão de representações, vamos chamar a atenção para as atividades cognitivas chamadas de formação, componente essencial das tarefas de produção. 
Segundo Duval (2009), a formação de uma representação semiótica é o recurso a um (ou a muitos) signo(s) de um sistema semiótico já constituído (a língua materna ou a língua simbólica, por exemplo), para designar um objeto. Ela "implica a seleção de certo número de caracteres de um conteúdo percebido, imaginado ou já representado em função de possibilidades de representação próprias ao registro escolhido" (DUVAL, 2009, p.56) e serve para exprimir uma representação mental ou evocar um objeto real. Por exemplo, para representar uma Integral Definida, são necessárias as unidades simbólicas $\int, a, b, f(x), d x$, para compor a unidade de nível superior $\int_{a}^{b} f(x) d x$ no Registro de Representação Algébrica.

Neste caso, podemos identificar os atos mais elementares de formação, que são, por exemplo, conforme os registros: a designação nominal "Integral Definida" e a codificação de relações como a expressão (1). Ou seja, a produção de uma representação semiótica está subordinada às regras sintáticas de formação e tratamento de unidades constitutivas de todas as representações possíveis num registro e que versam sobre:

$>$ a determinação de unidades elementares: símbolos, vocabulários etc.;

$>$ combinações admissíveis de unidades elementares para formar unidades de nível superior: como, por exemplo, regras de formação de um sistema formal, gramática da língua portuguesa, etc.;

as condições para que uma representação de ordem superior seja uma produção pertinente e completa.

A determinação destas unidades elementares está diretamente associada à atividade cognitiva de conversão de registros de representação semiótica, uma vez que o grau de correspondência associativa entre as unidades significantes elementares constitutivas de cada uma das duas representações pertencentes a dois registros diferentes, determina a facilidade ou não da conversão.

Quando há correspondência termo a termo entre as unidades significantes dos dois registros, dizemos que há congruência das representações e, neste caso, a conversão é quase imediata. Caso contrário, dizemos que as representações são não-congruentes. Nesse caso, a dificuldade de realizá-la depende do grau da não-congruência e da possibilidade de coordenação dos dois registros pelo indivíduo.

Sendo assim, estabelecer se duas representações entre dois registros diferentes são ou não congruentes implica, primeiramente, segundo Duval (2009), em segmentá-las em suas unidades significantes elementares respectivas, de forma tal que elas possam ser colocadas em correspondência. Ao final da comparação, é possível perceber se as unidades significantes são, em cada registro, simples ou combinação de unidades simples. 
Esta análise da congruência entre duas representações foi sintetizada por Duval (2009) nos três critérios de congruência:

1. Correspondência semântica das unidades significantes (CS): a cada unidade significante simples (elementar) de uma das representações, pode-se associar uma unidade significante elementar da outra representação.

2. Univocidade semântica terminal (US): a cada unidade significante elementar da representação de partida corresponde uma só unidade elementar no registro de representação de chegada (terminal).;

3. Mesma ordem possível de apreensão das unidades significantes nas duas representações $(\mathrm{OR})$.

Façamos, então, uma análise da congruência semântica envolvidas nas conversões de representação relacionadas ao somatório, convergência e integral definida como cálculo de área. Todos esses elementos que nos propusemos a analisar compõem o conceito de integral de Riemann e podem ser identificados em (1).

\subsection{A notação de somatório}

Uma simples tarefa de desenvolver a expressão $\sum_{i=1}^{3} i^{2}$, especificando seu valor numérico, requer uma conversão e um tratamento que transforma a representação inicial $\sum_{i=1}^{3} i^{2}$ na representação final 14 .

$$
\sum_{i=1}^{3} i^{2} \overbrace{=}^{\text {Conversão }} \underbrace{(1)^{2}}_{i=1}+\underbrace{(2)^{2}}_{i=2}+\underbrace{(3)^{2}}_{i=3} \overbrace{=}^{\text {Tratamento }} 1+4+9 \overbrace{=}^{\text {Tratamento }} 14
$$

A conversão é necessária para substituir o registro em forma de somatória (algébrica) em um registro numérico operacional (sob o ponto de vista aritmético). Em seguida, são necessários tratamentos que substituem os registros numéricos com as notações exponenciais nos registros numéricos $1+4+9$ e finalmente, outro tratamento, que substitui o registro numérico que indica a adição no registro numérico 14 .

Essa tarefa é, aparentemente, simples. Contudo, analisemos a primeira das operações a ser realizada pelo estudante ao resolver essa tarefa: a conversão. Segundo Brandt e Moretti:

\footnotetext{
A operação de conversão, por sua vez, não é nem trivial, nem cognitivamente neutra. A operação de conversão coloca tanto a questão do papel da semiósis no funcionamento do pensamento quanto o das condições de uma diferenciação entre representante e representado. A complexidade da conversão de representações só pode ser compreendida desde que se veja os sistemas semióticos em sua estreita relação com as representações ou mais exatamente ao par (conhecimento, representação) (BRANDT; MORETTI, 2005, p. 206).
} 
No quadro 1, é apresentada a análise de congruência semântica da conversão de representação algébrica $\sum_{i=1}^{3} i^{2}$ na representação numérica $1^{2}+2^{2}+3^{2}$, segundo os critérios estabelecidos por Duval.

Quadro 1: Análise de Congruência Semântica da conversão envolvida no desenvolvimento do somatório (xxxxxx,xxxx, p.188).

Tarefa: Analisar a congruência semântica da conversão da representação algébrica $\sum_{i=1}^{3} i^{2}$ na representação numérica $1^{2}+2^{2}+3^{2}$.

\begin{tabular}{|c|c|c|c|c|c|}
\hline \multirow{2}{*}{$\begin{array}{l}\text { Unidade } \\
\text { Significante do } \\
\text { registro de saída } \\
\text { (algébrico) }\end{array}$} & \multirow{2}{*}{$\begin{array}{c}\text { Unidade } \\
\text { Significante do } \\
\text { registro de chegada } \\
\text { (numérico) }\end{array}$} & \multicolumn{3}{|c|}{ Critérios de Congruência } & \multirow[b]{2}{*}{ Conclusão } \\
\hline & & $\mathrm{CS}$ & US & OR & \\
\hline$\Sigma$ & + & Sim & Sim & Não & \multirow{3}{*}{$\begin{array}{c}\text { Não } \\
\text { congruente }\end{array}$} \\
\hline$i^{2}$ & $2^{2}, \quad 3^{2}$ & Sim & Não & Não & \\
\hline$i$ & $1,2,3$ & Sim & Não & Sim & \\
\hline
\end{tabular}

A congruência semântica não tem a mesma dificuldade nos dois sentidos de conversão. O quadro 2 apresenta a análise de congruência semântica da mesma tarefa do quadro 1, mas desta vez no sentido numérico para algébrico.

Observe que a conversão no sentido numérico - algébrico (quadro 2) é mais difícil de ser realizada que no caso apresentado no quadro 1, embora ambas as conversões sejam não congruentes. Isso se dá pela quantidade de critérios de congruência não contemplados, o que está representado pela quantidade de "não" nos quadros 1 e 2.

Quadro 2: Análise de Congruência Semântica da conversão numérica para algébrica envolvida no desenvolvimento do somatório.

\begin{tabular}{|c|c|c|c|c|c|}
\hline \multicolumn{6}{|c|}{$\begin{array}{l}\text { Tarefa: Analisar a congruência semântica da conversão da representação numérica } \\
\qquad 1^{2}+2^{2}+3^{2} \text { na representação algébrica } \sum_{i=1}^{3} i^{2}\end{array}$} \\
\hline \multirow{2}{*}{$\begin{array}{c}\text { Unidade } \\
\text { Significante do } \\
\text { registro de saída } \\
\text { (numérico) }\end{array}$} & \multirow{2}{*}{$\begin{array}{c}\text { Unidade } \\
\text { Significante do } \\
\text { registro de chegada } \\
\text { (algébrico) }\end{array}$} & \multicolumn{3}{|c|}{ Critérios de Congruência } & \multirow[b]{2}{*}{ Conclusão } \\
\hline & & $\mathrm{CS}$ & US & OR & \\
\hline $1^{2}, \quad 2^{2}, \quad 3^{2}$ & $i^{2}$ & Sim & Não & Não & \multirow{3}{*}{$\begin{array}{l}\text { Não } \\
\text { congruente }\end{array}$} \\
\hline+ & $\Sigma$ & Sim & Não & Não & \\
\hline $1,2,3$ & $i$ & Sim & Não & Não & \\
\hline
\end{tabular}

Estas dificuldades na conversão puderam ser percebidas na resolução de uma atividade em que era dada uma expressão na forma concisa e o aluno deveria desenvolvê-la (tarefa 1 da figura 1) e vice-versa (tarefa 2 da figura 1). Embora as atividades da figura 1 não remetam, todas, à notação de somatório, a mesma análise de congruência é válida. 
Perceba que na tarefa 1 era exigida a conversão algébrica - numérica, enquanto a tarefa 2 requeria a conversão numérica-algébrica. A mesma dupla de estudantes teve maior dificuldade em realizar a tarefa 2 (note que foram dois erros em três itens) que na tarefa 1 (um erro em três itens).

Figura 1: dificuldades com o desenvolvimento de expressões na forma concisa e expandida (xxxxxx, p. 187).

Agora é com você!

1. Desenvolva as expressões seguintes:

a)

$3_{k=1}^{5}(3 k-5)\left(L_{k=1}^{5}(3 k-5)=(3.1-5)<(3.2-5)<(3.3-5) L(34.5)\right.$

b) $\bigcup_{m=2}^{5}\left(m^{2}\right)\left(C_{m=2}^{5}\left(m^{2}\right)=\left(1^{2}\right) C\left(1^{3}\right) C\left(1^{4}\right) C\left(1^{5}\right)\right.$

c) $\sum_{i=1}^{4}(2 i) \quad\left(\sum_{i=1}^{4}(2 i)=\left((2.1) \sum(2.2) \sum(2.3) \sum(2.4) \sum(2.5)\right.\right.$ 2. Agora, estão apresentados os desenvolvimentos das expressóes, sua tarefa é escrevê-las no
modo conciso.

a) $\quad(3 \cdot 5+7) \mathrm{O}_{(3 \cdot 6+7)} \mathrm{O}_{(3 \cdot 7+7)} \mathrm{O}_{(3 \cdot 8+7)} \mathrm{O}_{(3 \cdot 9+7)} \mathrm{O}_{(3 \cdot 10+7)}$

b) $1 \# \frac{1}{2} \# \frac{1}{3} \# \frac{1}{4} \# \cdots \# \frac{1}{10}$

c) $\left(\frac{2+1}{2-1}\right)^{2}=\left(\frac{3+1}{3-1}\right)^{3}=\left(\frac{4+1}{4-1}\right)^{4}=\left(\frac{5+1}{5-1}\right)^{5}=\ldots\left(\frac{10000+1}{10000-1}\right)^{10000}$

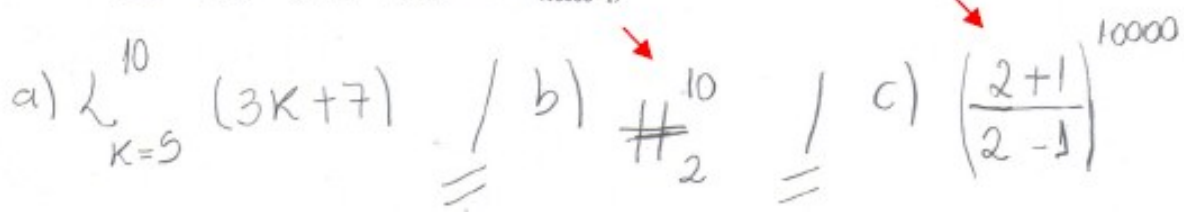

Ainda é possível associar a dificuldade na conversão analisada no quadro 2 à necessidade do estabelecimento de padrões, outro ponto que nem sempre é privilegiado na educação escolar.

A determinação de padrões é uma tarefa geralmente destinada aos matemáticos e exige uma postura investigativa do aluno. Entretanto, perceber padrões é importante para que o aluno compreenda os elementos componentes do conceito de integral definida.

Considere, por exemplo, a tarefa: escrever a sequência $\frac{2}{3}+\frac{1}{3}+\frac{1}{6}+\frac{1}{12}+\frac{1}{24}$ na forma concisa. Embora nesse processo seja usado basicamente o registro numérico, mesmo com a necessidade de mudança de representação dentro desse registro, o cumprimento da tarefa exige a análise dos padrões envolvidos em cada tratamento realizado, culminando numa conversão desse padrão para a representação algébrica, como indica a Figura 2. 
Figura 2: tratamentos e conversão envolvidos na determinação de um padrão (xxxxxxxxxxx, p.198)

$$
\begin{aligned}
& \frac{2}{3}+\frac{1}{3}+\frac{1}{6}+\frac{1}{12}+\frac{1}{24} \\
& 1 \text { Tratamento } \\
& \frac{2}{3}+\frac{1}{3}+\frac{1}{3} \cdot \frac{1}{2}+\frac{1}{3} \cdot \frac{1}{2} \cdot \frac{1}{2}+\frac{1}{3} \cdot \frac{1}{2} \cdot \frac{1}{2} \cdot \frac{1}{2} \\
& ]_{2} \text { Tratamento } \\
& \frac{2}{3}+\frac{1}{3}+\frac{1}{3} \cdot\left(\frac{1}{2}\right)^{1}+\frac{1}{3} \cdot\left(\frac{1}{2}\right)^{2}+\frac{1}{3} \cdot\left(\frac{1}{2}\right)^{3} \\
& { }_{3} \text { Tratamento } \\
& \frac{1}{3} \cdot\left(\frac{1}{2}\right)^{-1}+\frac{1}{3} \cdot\left(\frac{1}{2}\right)^{0}+\frac{1}{3} \cdot\left(\frac{1}{2}\right)^{1}+\frac{1}{3} \cdot\left(\frac{1}{2}\right)^{2}+\frac{1}{3} \cdot\left(\frac{1}{2}\right)^{3} \\
& 4 \text { Tratamento } \\
& \frac{1}{3} \cdot\left[\left(\frac{1}{2}\right)^{-1}+\left(\frac{1}{2}\right)^{0}+\left(\frac{1}{2}\right)^{1}+\left(\frac{1}{2}\right)^{2}+\left(\frac{1}{2}\right)^{3}\right] \\
& \sqrt{3} \\
& \text { Conversão } \\
& \frac{1}{3} \cdot \sum_{k=-1}^{3}\left(\frac{1}{2}\right)^{k} \\
& \frac{2}{3} \cdot \sum_{k=0}^{4}\left(\frac{1}{2}\right)^{k}
\end{aligned}
$$

\subsection{A convergência e a notação de limite}

Nem sempre fica claro ao estudante o significado da frase: um mesmo objeto pode ter diferentes representações. Quando se fala de sequências numéricas, vê-se claramente essa diversidade de representações. Considere, por exemplo, o objeto sequência numérica cujo termo geral é $a_{n}=\frac{2 n-1}{n}, n \in \mathbb{N}^{*}$. Um exemplo das várias representações desse objeto pode ser observado no Quadro 3. 
Quadro 3: Diferentes representações para uma sequência numérica

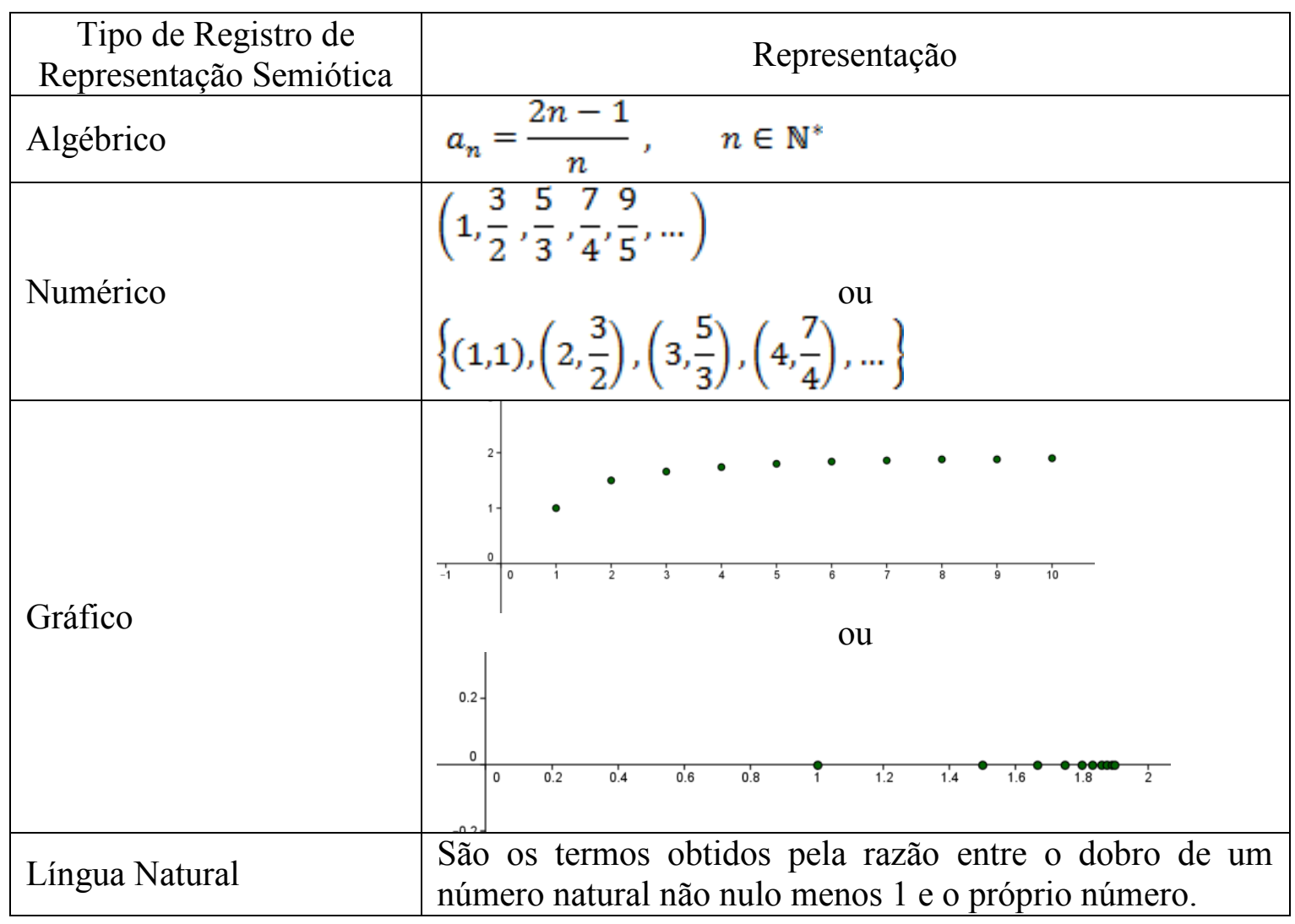

Observe que as representações diferem entre si, porém, emitem informações sobre o mesmo objeto matemático. Fazer com que o aluno compreenda que todas as representações apresentadas no Quadro 3 são faces da mesma moeda deve ser um dos objetivos do ensino. Entretanto, para que o trânsito espontâneo entre essas várias representações aconteça, é preciso atuar com a diversidade de Registros de Representação Semiótica e as tarefas de compreensão.

Imagine, por exemplo, a tarefa: analise o comportamento da sequência $a_{n}=\frac{1}{n}, n \in \mathbb{N}^{*}$ e represente algebricamente sua conclusão. Esta atividade é aparentemente simples, entretanto, para ser realizada, exige a conversão da representação em língua natural (RLN), indicada pela frase "analise o comportamento da sequência", para a representação algébrica (RA). É provável que o aluno use a representação gráfica $(R G)$ dessa sequência como apoio à conversão requerida. Nesse caso, é possível estabelecer como unidades significantes as que são mostradas no Quadro 4. 
Quadro 4: Unidades significantes para a conversão RLN $\rightarrow$ RA para a sequência $a_{n}=\frac{1}{n}, n \in \mathbb{N}^{*}$ (xxxxxx, p. 209).

\begin{tabular}{|c|c|c|c|}
\hline $\begin{array}{c}\text { Unidade Significante } \\
\text { Registro de Saída (RLN) }\end{array}$ & $\begin{array}{c}\text { Unidade Significante do "Registro } \\
\text { Intermediário" (RG) }\end{array}$ & $\begin{array}{c}\text { Unidade Significante } \\
\text { Registro de Chegada (RA) }\end{array}$ \\
\hline Aproximação a um ponto & $\bullet \quad \bullet \quad \bullet \quad \bullet \quad \bullet \quad \bullet \quad \bullet$ & $\lim _{n \rightarrow \infty} \frac{1}{n}=0$ \\
\hline
\end{tabular}

É importante observar que as operações cognitivas a serem realizadas pelo aluno nessa simples tarefa não tem nada de simples, mesmo porque ele dificilmente converterá as representações em língua natural para a algébrica sem passar por uma representação intermediária, seja ela a representação gráfica, como apresentada no quadro 4, ou a numérica. Isto dependerá da facilidade discente, que dependerá, provavelmente, do enfoque dado pelo ensino ao longo da sua escolarização.

Ao pretender que o estudante use a notação de limite para designar o comportamento de uma sequência, é necessário que o professor utilize desse processo de conversão e das tarefas de compreensão.

Na sequência didática aplicada no desenvolvimento da tese apresentada em Cargnin (2013), alunos que já tinham estudado a teoria de limites no curso regimental de Cálculo I não conseguiram usar a notação de limites para representar a convergência de uma sequência, o que indica a não compreensão adequada daquela teoria. A passagem da ideia de convergência para seu registro algébrico é difícil e cheio de não congruências, como mostra o Quadro 5.

No exemplo 1 do Quadro 5, pela análise dos critérios estabelecidos por Duval, a representação no registro em Língua Natural é congruente à representação algébrica. Já no exemplo 2, observamos a não congruência. Quanto mais "não" existirem nas colunas relativas aos critérios de convergência, maior é a dificuldade para o aluno fazer a conversão, pois haverá mais critérios de convergência não atendidos. Assim, exprimir a convergência usando a linguagem algébrica torna-se mais difícil para o estudante. Neste caso, é preciso mais tarefas de produção e compreensão para pleitear a compreensão necessária para este conceito. 
Quadro 5: Análise de Congruência para representações do conceito de convergência (xxxxxx, p.61)

\begin{tabular}{|c|c|c|c|c|c|c|}
\hline \multicolumn{7}{|c|}{$\begin{array}{l}\text { Exemplo 1: Verificar a congruência das representações nos registros língua natural (RLN) e } \\
\text { algébrico (RA) dadas por "a sequência } k_{n} \text { converge para o número } L " \text { e " } k_{n} \rightarrow L \text { ", } \\
\text { respectivamente. }\end{array}$} \\
\hline \multirow{2}{*}{$\begin{array}{l}\text { Unidades } \\
\text { Significantes } \\
\text { para RLN }\end{array}$} & \multirow{2}{*}{$\begin{array}{l}\text { Unidades } \\
\text { Significantes } \\
\text { para RA }\end{array}$} & \multirow{2}{*}{$\begin{array}{l}\text { Segmentação } \\
\text { Comparativa }\end{array}$} & \multicolumn{3}{|c|}{ Critério de Congruência } & \multirow{2}{*}{ Conclusão } \\
\hline & & & CS & US & OR & \\
\hline Sequência & $k_{n}$ & Simples & Sim & Sim & Sim & \multirow{3}{*}{ Congruente } \\
\hline Converge & $\rightarrow$ & Simples & Sim & Sim & Sim & \\
\hline $\mathrm{L}$ & $L$ & Simples & Sim & Sim & Sim & \\
\hline \multicolumn{7}{|c|}{$\begin{array}{l}\text { Exemplo 2: Verificar a congruência das representações nos registros língua natural (RLN) e } \\
\text { algébrico (RA) dadas por "a sequência } k_{n} \text { converge para o número } L " \mathrm{e} \text { " } \lim _{n \rightarrow \infty} k_{n}=L \text { " }\end{array}$} \\
\hline Sequência & $k_{n}$ & Simples & Sim & Sim & Não & \multirow{3}{*}{$\begin{array}{l}\text { Não } \\
\text { congruente }\end{array}$} \\
\hline Converge & $\lim , n, \rightarrow, \infty,=$ & Combinada & Sim & Não & Não & \\
\hline $\mathrm{L}$ & $\mathrm{L}$ & Simples & Sim & Sim & Sim & \\
\hline
\end{tabular}

A segmentação comparativa mostrada no Quadro 5 indica a existência, ou não, da univocidade semântica terminal (US). Quando a segmentação é comparada, não há congruência, mesmo que essa não-congruência seja leve.

Note ainda, no Quadro 5, que a notação usada para a convergência de uma sequência (ou série) nos cursos de Cálculo é a não congruente, e isso sem passar pela notação que é congruente.

Um modo de avançar no processo de ensino e aprendizagem das noções de convergência é explorar a significação do conceito usando a língua natural, cujos benefícios já foram explanados por Mariani (2006 ) e Dallanese ( 2006), entre outros.

Outra possibilidade para o melhor entendimento e incentivo ao discurso matemático é estimular os estudantes a conversarem sobre uma definição para tal e escreverem um critério de convergência usando o registro algébrico. Isto poderia facilitar a compreensão da definição formal, ao mesmo tempo em que o professor pode perceber as dificuldades de conceitos e símbolos que os alunos ainda têm.

A articulação dos registros visual e discursivo passa pela codificação das conexões locais, isto é, pela identificação das variáveis visuais do gráfico que correspondem às variáveis semânticas. Da mesma forma, a articulação dos registros discursivo e algébrico requer a correspondência de unidades semânticas e unidades simbólicas (DUVAL, 2006). 
Para tanto, é indispensável que o aluno seja estimulado a pensar globalmente acerca da atividade que lhe é proposta e procurar analisar as consequências que uma mudança ocorrida num determinado registro acarreta ao outro registro.

Do ponto de vista das Representações Semióticas, considere as unidades significantes para os registros em Língua Natural, Gráfico e Algébrico como apresentadas no Quadro 6, referente ao conceito de limite.

A afirmação "quando a abscissa tende ao infinito, a ordenada $k_{n}$ se aproxima do número L", não é semanticamente congruente ao registro algébrico $\lim _{n \rightarrow \infty} k_{n}=L$ (lida como $k_{n}$ se aproxima de $L$ quando n tende ao infinito), pois há uma inversão na ordem das unidades significantes correspondentes, o que aumenta o custo da operação cognitiva realizada pelo aluno. A mesma não congruência acontece se a expressão citada for lida como “o limite de $k_{n}$ é $L$ quando $n$ tende ao infinito".

Contudo, pode-se perceber que a primeira forma de leitura da expressão algébrica apresenta uma combinação das unidades semânticas que se aproxima mais da representação gráfica, enquanto a segunda, tem maior aproximação em relação a representação algébrica.

A segunda forma de leitura pode trazer consigo outros obstáculos, pois, além da inversão, contém uma igualdade implícita (é $L$ ). Tal igualdade é, na notação de limite, indistinta da igualdade aritmética trabalhada na escola desde os anos iniciais, mas ela é uma nova igualdade que tem o significado de aproximação, de tendência, conceitos que usam outros verbos para se consolidarem. Ou seja, o mesmo significante, o signo “=”, deve receber outra significação no estudo do cálculo. É esse o ponto no qual se passa de um conceito de infinito potencial a um conceito de infinito atual ou atingido, dominado pelos matemáticos mediante manipulação de signos como: épsilons e deltas.

No entanto, a afirmação "quando a abscissa tende ao infinito, a ordenada $k_{n}$ se aproxima do número L", é semanticamente congruente à afirmação " $n \rightarrow \infty \Rightarrow k_{n} \rightarrow L$ ", por atender aos critérios de congruência (CARGNIN, 2013, p.130-133). 
Quadro 6: Unidades Significantes para os registros associados ao conceito de limite (xxxxxx, p. 132).

\begin{tabular}{|c|c|c|}
\hline Tipo de Registro & Unidades Significantes & $\begin{array}{c}\text { Combinações admissíveis das unidades } \\
\text { significantes }\end{array}$ \\
\hline Lingua Natural & $\begin{array}{c}\text { (unidades semânticas) } \\
\text { Número } \\
\text { Tende a } \\
\text { Abscissa } \\
\text { Ordenada } \\
\text { Se aproxima de } \\
\text { Infinito } \\
\text { Sequência X } \\
\text { Convergente }\end{array}$ & $\begin{array}{l}\text { quando a abscissa tende ao infinito, a } \\
\text { ordenada } k_{n} \text { se aproxima do número } \mathrm{L} \text {. }\end{array}$ \\
\hline Gráfico & $\begin{array}{l}\text { (unidades visuais) } \\
\text { Assintota horizontal } \\
\text { Comportamento da } \\
\text { ordenada (tendência do } \\
\text { valor da ordenada) } \\
\text { Comportamento da abscissa } \\
\text { (tendência do valor da } \\
\text { abscissa) }\end{array}$ & $\begin{array}{l}\text { (considerou-se, como exemplo, a sequência } \\
k_{n}=\frac{L n-1}{n} \text { ) }\end{array}$ \\
\hline Algébrico & $\begin{array}{c}\text { (unidades simbólicas) } \\
\text { Lim } \\
a_{n} \\
\text { Número L } \\
n \text { (variável) } \\
\rightarrow \\
\infty\end{array}$ & $\lim _{n \rightarrow \infty} \boldsymbol{k}_{n}=L$ \\
\hline
\end{tabular}

Em particular, se considerarmos uma 'definição intuitiva' para sequência convergente dada por: "uma sequência numérica converge para um número L se os termos da sequência ficam cada vez mais próximos do número real L", não há congruência semântica entre os registros de representação algébrica e em língua natural, haja vista que os critérios de correspondência semântica das unidades significantes e univocidade semântica terminal não são satisfeitos, já que à expressão "termos da sequência", que pode ser considerada uma unidade significante para representação em língua natural, para a definição intuitiva em análise, correspondem as unidades significantes $n, k_{n},\left(n, k_{n}\right)$ do registro na representação algébrica. 
Já se considerarmos a definição formal de convergência: “A sequência $\left\{a_{n}\right\}$ converge para o número $L$ se para todo número positivo $\varepsilon$, existe um número inteiro $N$ tal que para todo $n, n>N \Rightarrow\left|a_{n}-L\right|<\varepsilon$. Se esse número $N$ não existe, dizemos que $\left\{a_{n}\right\}$ diverge", a dificuldade de compreensão para o aluno é ainda maior. Em parte, isso se deve ao uso de símbolos na definição, que normalmente acarreta incompreensão do significado de cada um deles no contexto da definição. Mas por outro lado, pode ser devido a não congruência entre as representações " $a_{n}$ se aproxima do número $L$ ” " $\left|a_{n}-L\right|<\varepsilon$ ”, como mostrado no Quadro 7.

Quadro 7: Análise de Congruência RLN $\rightarrow$ RA (xxxxxxxx, p.145).

Análise de congruência das representações nos registros língua natural (RLN) e algébrico (RA) dadas por " $a_{n}$ se aproxima do número $L$ " e " $\left|a_{n}-L\right|<\varepsilon$ ", respectivamente.

\begin{tabular}{|c|c|c|c|c|c|c|}
\hline \multirow{2}{*}{$\begin{array}{l}\text { Unidades } \\
\text { Significantes } \\
\text { para RLN }\end{array}$} & \multirow{2}{*}{$\begin{array}{l}\text { Unidades } \\
\text { Significantes } \\
\text { para RA }\end{array}$} & \multirow{2}{*}{$\begin{array}{l}\text { Segmentação } \\
\text { Comparativa }\end{array}$} & \multicolumn{3}{|c|}{ Critérios de Congruência } & \multirow[b]{2}{*}{ Conclusão } \\
\hline & & & CS & US & OR & \\
\hline$a_{n}$ & $a_{n}$ & Simples & Sim & Sim & Não & \multirow{3}{*}{$\begin{array}{c}\text { Não } \\
\text { congruente }\end{array}$} \\
\hline Aproxima & $\mid,-,<, \varepsilon$ & Combinada & Sim & Não & Não & \\
\hline$L$ & $L$ & Simples & Sim & Sim & Não & \\
\hline
\end{tabular}

Nem sempre os alunos do ensino superior conseguem atribuir um significado ao significante $\left|a_{n}-L\right|<\varepsilon$, para $n>N$ dado. Nesse caso, não sendo possível estabelecer uma significação ao significante, o aluno torna-se incapaz de compreender que a expressão designa a proximidade de pontos de uma sequência em relação ao seu valor limite, e está associada à

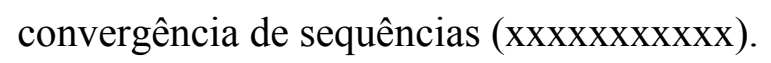

\subsection{A Integral Definida como cálculo de área}

Quando consideramos o conceito de integral definida, intrínseco a ele, por sua própria definição, estão as dificuldades já apontadas nos itens 1.1 e 1.2. No entanto, ao usar a integral definida para o cálculo da área de uma região limitada surgem outros problemas.

Considere, por exemplo, a tarefa "Calcular a área sob a curva $x^{2}+1$ no intervalo $[1,3]$ ”. Essa tarefa contempla tratamentos e conversões, como indicado na Figura 3. As flechas 1 e 3 representam conversões da representação em língua natural para as representações algébrica e gráfica, respectivamente. As flechas 2 e 4 representam conversões da representação algébrica em gráfica e vice-versa, respectivamente. A flecha 5 indica o tratamento para o cálculo da área no registro algébrico. 
Nessa tarefa, é possível ainda um tratamento gráfico para o cálculo da área, por meio da aproximação com soma de áreas de $n$ retângulos, como indicado na Figura 4.

Figura 3: Exemplo de tratamento e conversão no cálculo de área (xxxxxx, p.56)

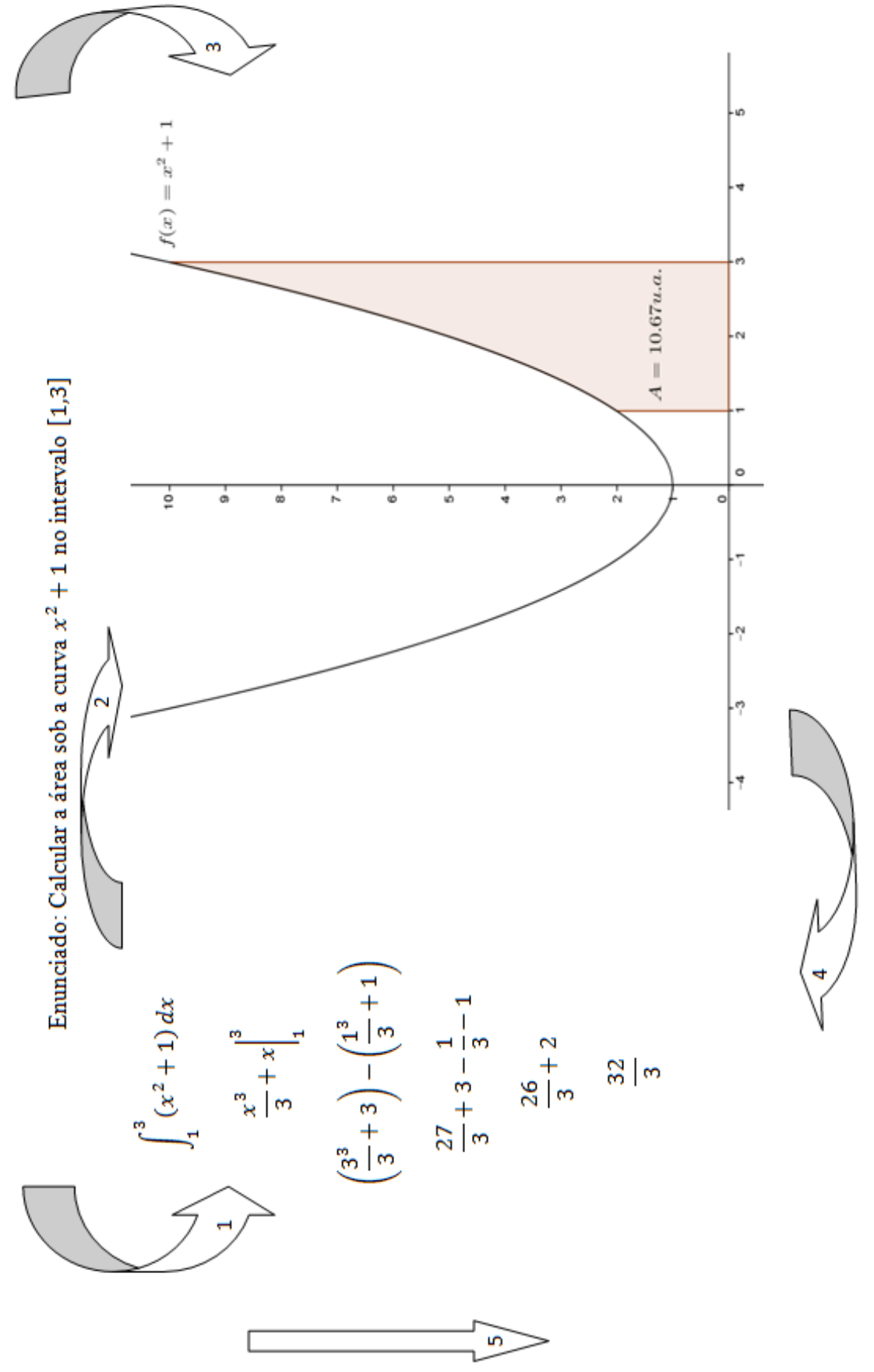


Figura 4: Tratamento gráfico para o cálculo de área sob uma curva (xxxxxxx, p.57).

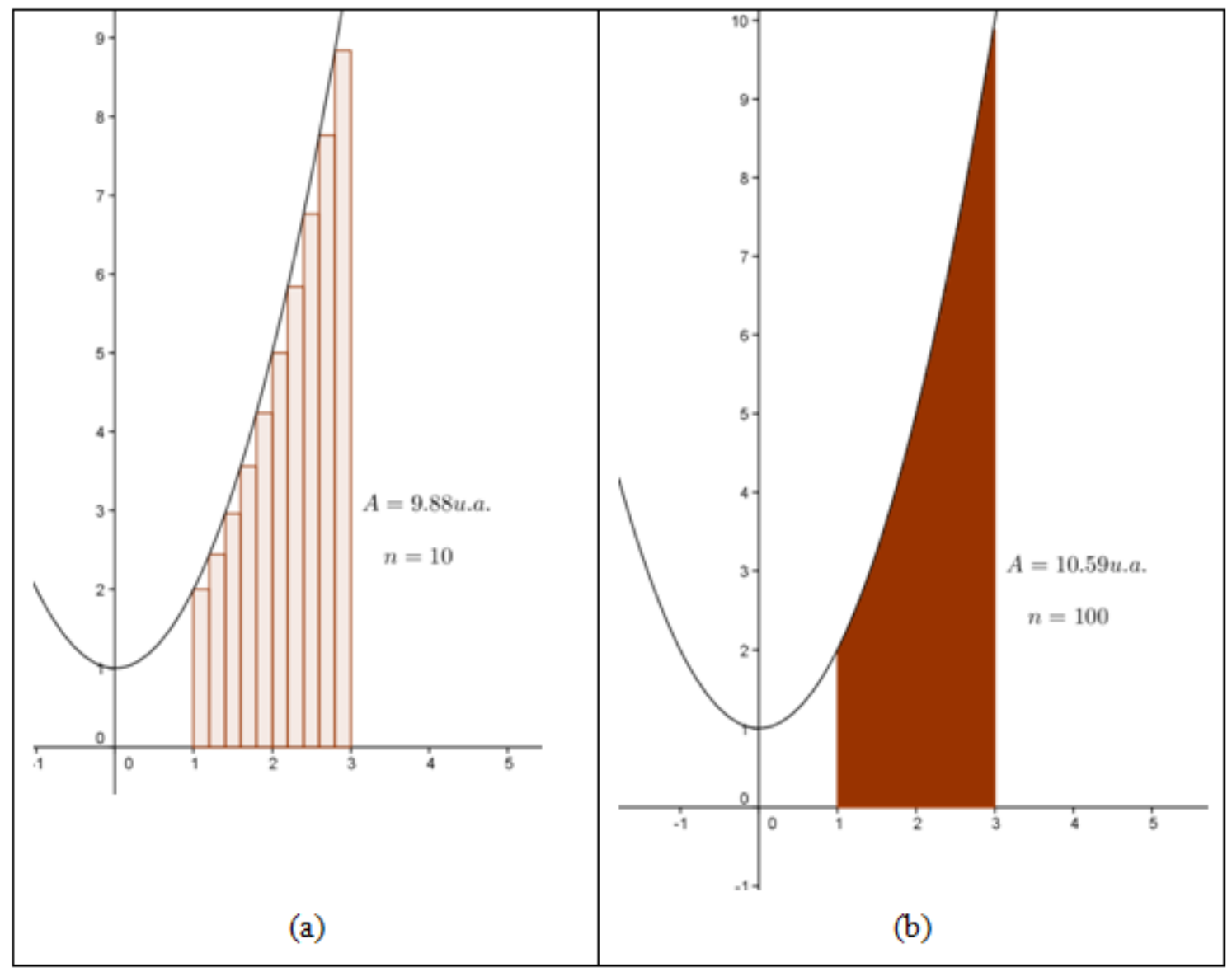

Analisemos, agora, a congruência entre as representações envolvidas na conversão 1 (flecha 1) da Figura 3, que trata de converter a representação no registro em Língua Natural "Calcular a área sob a curva $x^{2}+1$, no intervalo [1,3]" na representação no Registro Algébrico “ $\int_{a}^{b}\left(x^{2}+1\right) d x "$. As unidades significantes elementares de cada representação e os critérios de congruência são apresentadas no Quadro 8.

Aqui, verifica-se a segmentação comparativa combinada para as unidades significantes dos registros RLN e RA, o que já implica em não univocidade semântica terminal, um dos fatores de não congruência. 
Quadro 8: Análise de congruência para representações do conceito do cálculo de área por meio de integrais (xxxxx, p.62).

\begin{tabular}{|c|c|c|c|c|c|c|}
\hline \multicolumn{7}{|c|}{$\begin{array}{l}\text { Verificar a congruência das representações nos RLN e RA, dadas por "Calcular a área sob a } \\
\text { curva } x^{2}+1 \text {, no intervalo }[1,3] " \text { e " } \int_{a}^{b}\left(x^{2}+1\right) d x \text { onde } a=1 \text { e } b=3 \text { ", respectivamente. }\end{array}$} \\
\hline \multirow{2}{*}{$\begin{array}{c}\text { Unidades } \\
\text { Significantes } \\
\text { para } R L N\end{array}$} & \multirow{2}{*}{$\begin{array}{c}\text { Unidades } \\
\text { Significantes } \\
\text { para RA }\end{array}$} & \multirow{2}{*}{$\begin{array}{l}\text { Segmentação } \\
\text { Comparativa }\end{array}$} & \multicolumn{3}{|c|}{ Critério de Congruência } & \multirow{2}{*}{ Conclusão } \\
\hline & & & CS & US & OR & \\
\hline Área & $\begin{array}{c}\int_{a}^{b} \mathrm{dx}, \text { para } \\
a \text { e } b \\
\text { definidos }\end{array}$ & Combinada & Sim & Não & Sim & \multirow{3}{*}{$\begin{array}{l}\text { Não } \\
\text { congruente }\end{array}$} \\
\hline Sob a Curva & $x^{2}+1$ & Combinada & Sim & Não & $\operatorname{Sim}$ & \\
\hline Intervalo $[1,3]$ & $\begin{array}{c}\int_{a}^{b}, \text { onde } \\
a=1 \text { e } b=3\end{array}$ & Combinada & Sim & Não & Não & \\
\hline
\end{tabular}

Quando a conversão em questão envolve um Registro Gráfico, há algumas outras questões a serem observadas. Isto porque, quando se trata de imagens, a representação semiótica é analógica, ou seja, conserva as propriedades do objeto, diferente do que acontece nas representações não-analógicas, já que, embora não conservem as propriedades, podem permitir operações ou transformações sobre o modelo (DUVAL, 2009).

Segundo Duval (2012), nas atividades matemáticas, as figuras são objeto de duas atitudes geralmente contrárias. Uma é imediata e automática: a apreensão perceptiva das formas. A outra é controlada: a interpretação discursiva (isto é, comandada pelas hipóteses do enunciado) dos elementos figurais. É esta segunda atitude que deveria prevalecer no ensino.

Toda figura pode ser modificada de modo que ela se torne uma subfigura por meio de alguma transformação como rotação, ou uma deformação, ou de modo que ela seja dividida em subfiguras. Essas modificações são chamadas, por Duval (2012), de modificações mereológicas.

As modificações mereológicas fazem surgir formas como um todo fracionado em partes homogêneas, ou seja, a forma da parte é a mesma do todo, ou em partes heterogêneas, neste caso, todo e partes não tem a mesma forma.

Desse fracionamento origina-se a operação de reconfiguração intermediária, que pode fazer com que as partes elementares sejam reagrupadas em várias subfiguras, todas pertencendo à mesma figura inicial.

Essa operação se faz presente ao se determinar a área sob uma curva, por exemplo, uma das possíveis aplicações da Integral Definida. Na Figura 5, a área sob a curva 
$c(x)=x^{4}+x+1$ no intervalo $[-1,1]$ é fracionada em retângulos, cuja soma das respectivas áreas $(2,26$ u. a. $)$ é uma aproximação para a área sob a curva, no intervalo pedido.

Figura 5: área sob uma curva, calculada por reconfiguração intermediária (xxxxx, p.63)

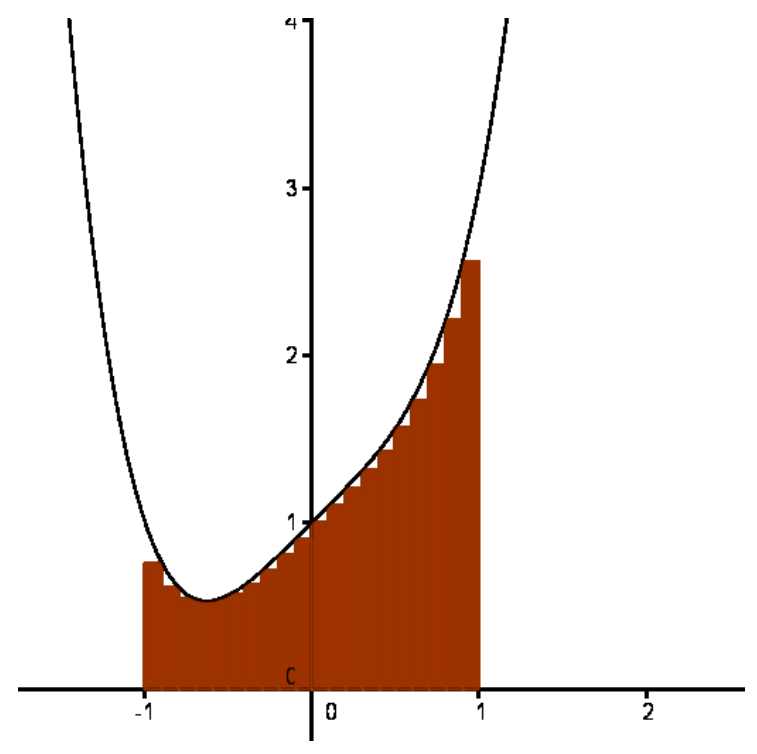

O fracionamento mostrado na Figura 5 não é o único possível. Cada aluno pode fracionar a região de modo diferente, dependendo de sua apreensão perceptiva. A Figura 6 mostra o fracionamento por trapézios. Contudo, no fracionamento por retângulos, idéia usualmente utilizada para que o aluno compreenda a fórmula utilizada na definição da integral de Riemann (expressão (1)) e calcule a área sob uma curva limitada, quanto maior a quantidade de retângulos, melhor será a aproximação.

Figura 6: Fracionamento de uma região por trapézios

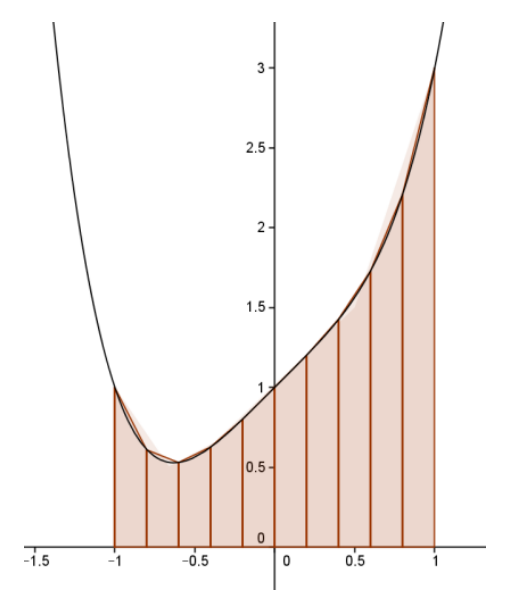

$\mathrm{Na}$ atividade de conversão que envolve uma representação gráfica, a determinação de unidades visuais é essencial, porém, isso depende da apreensão perceptiva discente da figura/gráfico e do tratamento dado a essas representações durante a escolarização. 
Nesse processo de conversão, a coordenação de registros de representação implica conhecer cada tipo de representação para saber correlacioná-las e, posteriormente, convertêlas. Porém, essa é uma tarefa que não pode ficar apenas sob a responsabilidade do aluno. É preciso que o trabalho desenvolvido em sala de aula seja também nesse sentido.

\section{Considerações Finais}

XXXX expõe a maneira simplista com que alguns livros de Cálculo tem apresentado o conceito de Integral Definida. Nesse artigo, pretendemos mostrar que o conceito em tela está repleto de não congruências implícitas, o que acarreta, segundo a teoria de Registro de Representação Semiótica, um maior custo cognitivo ao aluno aprendente.

As não-congruências apresentadas evidenciam a necessidade de maior cuidado com a introdução desse conceito. É certo que os recursos computacionais atuais ainda são fonte de benefícios e exploração discente e trazem grandes contribuições à aprendizagem. Porém, é preciso mais que apenas usar um recurso computacional para que o aluno de cálculo I entenda o processo que se pretende ensinar e possa usar o conceito estudado em outros conceitos.

Ao se trabalhar no ensino apenas com um tipo de registro semiótico, a aprendizagem pode ficar prejudicada, justamente pela significação que o estudante atribui ao que está sendo estudado. Ao usar mais registros de representação semiótica, com tratamentos e conversões entre essas representações, é possível contribuir com a conceitualização pretendida.

Atividades aplicadas a alunos durante a pesquisa apresentada em xxxxxx indicam que o uso das representações gráficas reforça o interesse e a análise dos conceitos estudados. Contudo, percebeu-se a necessidade de uma abordagem global dessas representações e não apenas pontual.

Ao usar diversos registros de representação semiótica, especialmente a língua natural, o professor pode diminuir a dificuldade discente em expressar as percepções sobre os experimentos (uma análise gráfica, por exemplo) em registros algébricos formais, assim como, em compreender as notações usadas nas definiç̧ões. Com isso também é possível que o docente perceba se aluno e professor atribuem a mesma significação ao conceito em estudo.

O professor possui um importante papel para que seu aluno obtenha sucesso na aprendizagem, uma vez que ele precisa contemplar uma adequação do conhecimento a ser adquirido em situações adidáticas, além de prever possíveis obstáculos a serem superados. Para isso, estar atento às dificuldades intrínsecas a cada conceito é fundamental e, para alguns, exige uma nova postura, mais investigativa, menos controlada e, talvez, mais eficaz. 


\section{Referências:}

BARROSO, N. M. C. et al. Uma sequência de ensino para a introdução do conceito de integral de Riemann. Anais IX ENEM, 2008. Disponível em www.sbem.com.br/files/ix_enem/Comunicacao_Cientifica/Trabalhos/CC23187786391T.doc Acesso em 24 jan. 2012.

BRANDT, C.F.; MORETTI, M.T. O papel dos registros de representação na compreensão do sistema de numeração decimal. Educação Matemática Pesquisa. São Paulo, 7(2), pp. 201-227, 2005. Disponível em http://revistas.pucsp.br/index.php/emp/article/view/4701/3269 Acesso em 16 fev. 2013.

DALL'ANESE, C. Argumentos e Metáforas conceituais para a taxa de variação. 2006. 131p. Tese (Doutorado em Educação Matemática). Pontifícia Universidade Católica de São Paulo- PUCSP, São Paulo, 2006. Disponível em http://www.pucsp.br/pos/edmat/do/tese/claudio_dallanese.pdf Acesso em 21 abr. 2011.

DUVAL, R. Abordagem Cognitiva de problemas de geometria em termos de congruência. Trad. Méricles T. Moretti. Revemat: R.Eletr. de Edu. Mat. Florianópolis (SC), 7(1), pp.118-138, 2012.

DUVAL, R. Semiósis e Pensamento Humano: Registros semióticos e aprendizagens intelectuais. (fascículo I). Tradução de Lenio Fernandes Levy e Marisa Rosâni Abreu da Silveira. São Paulo: Editora Livraria da Física, 2009.

DUVAL, R. Un tema crucial en la educación matemática: La habilidad para cambiar el registro de representación. La Gaceta de La RSME, v.9.1, 2006, pp.143-168.

MARIANI, R. C. P. Transição da Educação Básica para o Ensino Superior: coordenação de registros de representação e os conhecimentos mobilizados pelos alunos no curso de Cálculo. 2006. 233p. Tese (doutorado em educação Matemática). Pontifícia Universidade Católica de São Paulo- PUCSP, São Paulo, 2009. Disponível em http://www.pucsp.br/pos/edmat/do/tese/rita_cassia_pistoia_mariani.pdf Acesso em 22 abr. 2011.

MELO, J. M. R. Conceito de Integral: uma proposta computacional para seu ensino e aprendizagem. Dissertação. (Programa de Pós-Graduação em Educação Matemática). Pontifícia Universidade Católica de São Paulo, São Paulo, 2002. Disponível em http://www4.pucsp.br/pos/edmat/ma/dissertacao/jose_manuel_melo.pdf Acesso em 14 jan.2012.

MORETTI, M. T. O papel dos registros de representação na aprendizagem de matemática. Contrapontos. Ano 2, n.6, p.423-437, Itajaí, set/dez 2002. Disponível em http://www6.univali.br/seer/index.php/rc/article/viewFile/180/152. Acesso em 20 jul.12. 